\title{
Block Forward Backward method for Computation of Electromagnetic Wave scattering from a collection of Inhomogeneous Bodies
}

\author{
Bijilash Babu, Conor Brennan and Marissa Condon *
}

\begin{abstract}
A Block Forward Backward (BFB) method is described for the computation of electromagnetic fields scattered from a collection of inhomogeneous bodies. The electric field volume integral equation is used to formulate the problem. It is discretised using the method of moments and a discrete FFT is used to accelerate the matrix vector multiplications at the heart of the BFB method. Numerical results confirm that the convergence rate achieved can be greatly in excess of that obtained using Krylov methods.
\end{abstract}

\section{INTRODUCTION}

The integral equation formulation of electromagnetic wave scattering problems offers the potential for machine level accuracy but suffers from high computational complexity. This is due to the need to solve a dense linear system in order to compute the amplitudes of the basis functions, situated on the scatterer surface, or in the case of inhomogeneous bodies, throughout the scatterer volume. Once found these amplitudes can be used to compute the fields throughout the problem region. The need to use many basis functions to adequately represent the fast-varying phase of the fields leads to linear systems of huge order that cannot be readily inverted (or stored). Instead practitioners use iterative methods which sequentially refine an estimate of the unknown fields until some accuracy threshold is met. Iterative methods based on Krylov subspaces are popular, due to to their robust convergence properties. However, recent work has suggested that stationary iterative methods such as those based on Gauss-Seidel (GS) or Successive Symmetric Over-Relaxation (SSOR) iteration can outperform the Krylov solvers in certain situations $[1,2,3,4]$. The latter method (with relaxation parameter $\omega$ set to 1 ) is commonly referred to as "forward-backward" (FB) in the Electromagnetics literature, and we shall refer to it as the forward-backward method throughout this paper. Being based on matrix multiplications these stationary methods can also be accelerated by proper

*Research Institute for Networks and Communications Engineeering (RINCE), School of Electronic Engineering, Dublin City University, Dublin 9, Ireland e-mail: brennanc@eeng.dcu.ie, tel.: +3531700 7649, fax: +353 17005508 . incorporation of the Fast Fourier Transform (FFT) when appropriate. This paper outlines the application of block stationary iterative solvers to the problem of scattering from a collection of inhomogeneous bodies embedded in a homogeneous background medium.

\section{VOLUME ELECTRIC FIELD INTE- GRAL EQUATION}

We assume a collection of two-dimensional inhomogeneous bodies embedded in an infinite homogeneous background medium with electrical parameters $\left(\epsilon_{0}, \mu_{0}\right)$. The bodies are assumed infinite in the $z$ direction. The relative permittivity $\epsilon_{r}(\mathbf{r})$ is assumed to vary from point to point within each body. A source radiating with time variation $\exp (\jmath \omega t)$ produces an incident field which interacts with the bodies to produce a scattered field which can be expressed as a volume integral convolving the equivalent polarization currents at each point,

$$
J_{z}(\mathbf{r})=\jmath \omega \epsilon_{0}\left(\epsilon_{r}(\mathbf{r})-1\right) E_{z}(\mathbf{r}),
$$

with a Green's function. The resultant integral equation is [5]

$$
E_{z}^{\text {inc }}(\mathbf{r})=\frac{J_{z}}{\jmath \omega \epsilon_{0}\left(\epsilon_{r}-1\right)}+\jmath \omega \mu_{0} A_{z}(\mathbf{r}),
$$

with

$$
A_{z}(\mathbf{r})=\iint J_{z}\left(\mathbf{r}^{\prime}\right) \frac{1}{4 \jmath} H_{0}^{(2)}\left(k_{0}\left|\mathbf{r}-\mathbf{r}^{\prime}\right|\right) d S^{\prime} .
$$

Solving for the currents allows us to compute the scattered fields (and hence total fields) everywhere in space. The problem is discretised by introducing $N$ pulse basis functions (defined on identical square cells) and point matching at the cell centres. This leads to a dense matrix equation of order $N$.

$$
\mathbf{Z} \mathbf{J}=\mathbf{V} \text {. }
$$

The matrix and right hand side vector elements are given by [5]

$$
\begin{aligned}
Z_{i j} & =\kappa J_{1}\left(k_{0} a_{j}\right) H_{0}^{(2)}\left(k_{0} R_{i j}\right) \\
Z_{i i} & =\kappa H_{1}^{(2)}\left(k_{0} a_{i}\right)-\frac{J \eta_{0} \epsilon_{r}\left(\mathbf{r}_{i}\right)}{k_{0}\left(\epsilon_{r}\left(\mathbf{r}_{i}\right)-1\right)}, \\
V_{i} & =E_{z}\left(\mathbf{r}_{i}\right) .
\end{aligned}
$$


where $R_{i j}$ is the distance between cells $i$ and $j$, $\kappa=\left(\pi \eta_{0} a_{e}\right) / 2$ and $\eta_{0}$ and $k_{0}$ are the impedance and wave-number of the background medium respectively. $a_{j}$ is the radius of a circle of equal area to cell $j$.

\section{BLOCK STATIONARY ITERATIVE SOLVERS}

Gauss-Seidel iteration [6] solves a matrix equation by sequentially updating the unknowns $J_{i}$ in the order $i=1 \cdots N$, according to

$$
Z_{i i} J_{i}^{(k+1)}=V_{i}-A_{i}^{(k+1)}-B_{i}^{(k)} \text { for } i=1 \cdots N,
$$

where

$$
\begin{aligned}
A_{i}^{(k)} & =\sum_{j<i} Z_{i j} J_{j}^{(k)}, \\
B_{i}^{(k)} & =\sum_{j>i} Z_{i j} J_{j}^{(k)} .
\end{aligned}
$$

The convergence of the algorithm can be investigated in terms of how the residual error defined by

$$
\boldsymbol{\epsilon}^{(k)}=\mathbf{J}^{(k)}-\mathbf{J},
$$

evolves from iteration to iteration. Decomposing $\mathbf{Z}$ into its diagonal $\mathbf{D}$, lower triangular $\mathbf{L}$ and uppertriangular $\mathbf{U}$ parts

$$
\mathbf{Z}=\mathbf{D}+\mathbf{L}+\mathbf{U},
$$

it is possible to show that

$$
\begin{aligned}
\boldsymbol{\epsilon}^{(k+1)} & =(\mathbf{D}+\mathbf{L})^{-1} \mathbf{U} \boldsymbol{\epsilon}^{(k)} \\
& =\mathbf{M}^{G S} \boldsymbol{\epsilon}^{(k)}
\end{aligned}
$$

The algorithm will converge if the spectral radius of the iteration matrix $\mathbf{M}^{G S}$ is less than one. Forward-backward (SSOR with relaxation parameter $\omega$ set to 1) augments each forward sweep with a backward sweep to yield

$$
\begin{aligned}
Z_{i i} J_{i}^{\left(k+\frac{1}{2}\right)}= & V_{i}-A_{i}^{\left(k+\frac{1}{2}\right)}-B_{i}^{(k)}, \\
& \text { for } i=1 \cdots N, \\
Z_{i i} J_{i}^{(k+1)}= & V_{i}-A_{i}^{\left(k+\frac{1}{2}\right)}-B_{i}^{(k+1)} \\
& \text { for } i=N \cdots 1 .
\end{aligned}
$$

By convention one iteration is assumed to consist of one forward sweep (half-iteration) followed by one backward sweep (second half-iteration). The error evolves with iteration as

$$
\begin{aligned}
\boldsymbol{\epsilon}^{(k+1)} & =(\mathbf{D}+\mathbf{U})^{-1} \mathbf{L}(\mathbf{D}+\mathbf{L})^{-1} \mathbf{U} \boldsymbol{\epsilon}^{(k)},(1) \\
& =\mathbf{M}^{F B} \boldsymbol{\epsilon}^{(k)}
\end{aligned}
$$

In this work we instead apply block versions of the GS and FB algorithms to problems involving $M$ distinct inhomogeneous bodies. Let the diagonal sub-block of the impedance matrix containing interactions between basis functions in the $i^{t h}$ body and basis functions in the $j^{\text {th }}$ body be denoted by $\tilde{\mathbf{Z}}_{i j}$, while the unknowns and incident field in the $i^{t h}$ body are given by $\tilde{\mathbf{J}}_{i}$ and $\tilde{\mathbf{V}}_{i}$ respectively. The block FB (BFB) scheme updates the currents block by block according to

$$
\begin{aligned}
\tilde{\mathbf{J}}_{i}^{\left(k+\frac{1}{2}\right)}= & \tilde{\mathbf{Z}}_{i i}^{-1}\left(\tilde{\mathbf{V}}_{i}-\tilde{\mathbf{A}}_{i}^{\left(k+\frac{1}{2}\right)}-\tilde{\mathbf{B}}_{i}^{(k)}\right) \\
& \text { for } i=1 \cdots M, \\
\tilde{\mathbf{J}}_{i}^{(k+1)}= & \tilde{\mathbf{Z}}_{i i}^{-1}\left(\tilde{\mathbf{V}}_{i}-\tilde{\mathbf{A}}_{i}^{\left(k+\frac{1}{2}\right)}-\tilde{\mathbf{B}}_{i}^{(k+1)}\right) \\
& \text { for } i=M \cdots 1,
\end{aligned}
$$

where

$$
\begin{aligned}
\tilde{\mathbf{A}}_{i}^{(k)} & =\sum_{j<i} \tilde{\mathbf{Z}}_{i j} \tilde{\mathbf{J}}_{j}^{(k)}, \\
\tilde{\mathbf{B}}_{i}^{(k)} & =\sum_{j>i} \tilde{\mathbf{Z}}_{i j} \tilde{\mathbf{J}}_{j}^{(k)} .
\end{aligned}
$$

It is possible to show that the block FB method outlined in equations (19) and (20) are equivalent to performing the standard FB (equations (15) and (16)) to the preconditioned system

$$
\mathbf{P Z J}=\mathbf{P V}
$$

where $\mathbf{P}$ is a block diagonal matrix. The diagonal blocks of $\mathbf{P}$ are given by $\tilde{\mathbf{Z}}_{i i}^{-1}$ for $i=1 \cdots M$. Let $\mathbf{Z}_{p}=\mathbf{P Z}$ which has lower triangular, upper triangular and diagonal parts given by $\mathbf{L}_{p}, \mathbf{U}_{p}$ and $\mathbf{D}_{p}$. The convergence thus depends on

$$
\boldsymbol{\epsilon}^{(k+1)}=\mathbf{M}^{B F B} \boldsymbol{\epsilon}^{(k)},
$$

where the iteration matrix for block FB is given by

$$
\mathbf{M}^{B F B}=\left(\mathbf{D}_{p}+\mathbf{U}_{p}\right)^{-1} \mathbf{L}_{p}\left(\mathbf{D}_{p}+\mathbf{L}_{p}\right)^{-1} \mathbf{U}_{p} .
$$

The iteration matrix for the block Gauss-Seidel method is

$$
\mathbf{M}^{B G S}=\left(\mathbf{D}_{p}+\mathbf{L}_{p}\right)^{-1} \mathbf{U}_{p} .
$$

\section{IMPLEMENTATION}

Each sweep of the block algorithms involves the computation of matrix vector multiplications of the form

$$
\tilde{\mathbf{Z}}_{i j} \tilde{\mathbf{J}}_{j}^{(k)},
$$

for $i \neq j$. From the definition of the matrix entries and assuming a regular rectangular grid we see that 
each $\tilde{\mathbf{Z}}_{i j}$ is a Toeplitz block Toeplitz matrix and as such the multiplication of it and a vector can be efficiently computed using the 2D FFT [5]. In addition each step of the block algorithms requires that we pre-multiply a vector by $\tilde{\mathbf{Z}}_{i i}^{-1}$ in equations (15) and (16). These inverses are pre-computed, stored to the hard disk and read in as required. This large amount of pre-computing is justified in that once stored they can be re-used for many problem configurations involving different source locations.

\section{RESULTS}

The first problem consists of four square inhomogeneous scatterers of side $\lambda_{0} / 2$ (at $f=300 \mathrm{MHz}$ ) arranged in a row and separated by gaps of $1 \lambda$. The incident field is a unit amplitude plane wave travelling in the $-\hat{\jmath}$ direction. Each scatterer is divided into 16 rows of 16 cells ( 256 cells in each body, yielding 1024 unknowns in total). A nominal value of $\epsilon_{r}=4$ was used for each square and each cell was given a random value for $\epsilon_{r}$ within $10 \%$ of this value, that is

$$
\epsilon_{r}(\mathbf{r})=(1.0+0.2 *(r-0.5)) * 4.0
$$

where $r$ is a random variable uniformly distributed on the range $[0,1]$. Figure 1 displays the logarithm of the absolute values of the eigenvalues of the $\mathbf{M}^{B G S}$ and $\mathbf{M}^{B F B}$ iteration matrices. We note that the spectral radius in both cases is less than one, guaranteeing convergence for this problem. Figure 2 compares the convergence rates of the block forward-backward, block Gauss-Seidel and a preconditioned conjugate gradient applied to the normal equations (CGNE)[5]. The CGNE applies the standard Conjugate Gradient method to the equation

$$
\mathbf{Z}^{\dagger} \mathbf{Z J}=\mathbf{Z}^{\dagger} \mathbf{V}
$$

where pre-multiplication by the conjugate transpose $\mathbf{Z}^{\dagger}$ ensures that the system on the left hand side is Hermitian positive definite. The preconditioner used was the matrix $\mathbf{P}$ as described in section 3. Figure 2 plots the logarithm of the normalised error $\delta^{(k)}$ against iteration number for each method where

$$
\delta^{(k)}=\frac{\left\|\mathbf{Z} \mathbf{J}^{(k)}-\mathbf{V}\right\|_{2}}{\|\mathbf{V}\|_{2}}
$$

It is seen that both the stationary block methods lead to convergent results in fewer iterations than the preconditioned CGNE. The BGS error reaches machine precision in roughly 70 iterations, while BFB reaches the threshold at around 20 (equivalent to roughly 40 BGS iterations). The computational advantage is all the greater when one considers that the computational cost of each step of the stationary methods is less than that of the preconditioned CGNE.

The second problem involves 12 bodies of side $\lambda_{0}(f=300 \mathrm{MHz})$ arranged in three rows of 4 , each separated by $5 \lambda_{0}$. In this case the total number of unknowns is equal to 12288 . The permittivity values are chosen as in the last example and the incident wave was the same. Figure 3 shows the log of the error for the block Gauss-Seidel and block forward-backward methods for this example. Again, they display rapid convergence, reaching machine precision in under 40 and under 20 iterations respectively.

It should be noted that the algorithm was also applied to bodies which were closer together than in the two examples given. In these cases the results were mixed, and in many cases the block stationary methods diverged. Research is ongoing for these cases to see how the algorithms' behaviour can be improved in these cases. The incorporation of a buffer zone such as is described in [2] and the use of relaxation parameters are two ideas being investigated.

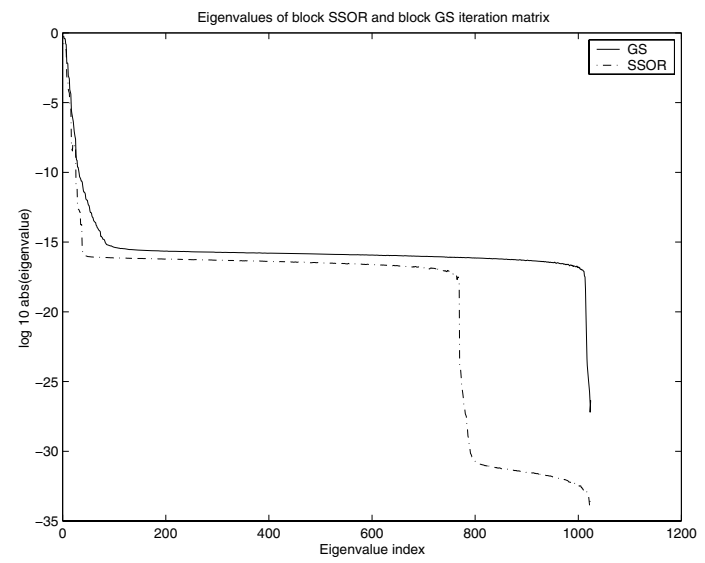

Figure 1: Eigenvalues of the iteration matrices

\section{CONCLUSIONS}

Numerical results have been presented illustrating the application of block stationary iterative methods to the computation of wave scattering from a collection of inhomogeneous bodies. The stationary methods are shown to produce convergent results with fewer iterations than the CGNE a Krylovsubspace based solver. Future work will concentrate on modification of the stationary methods to ensure convergence in situations where it presently diverges. 


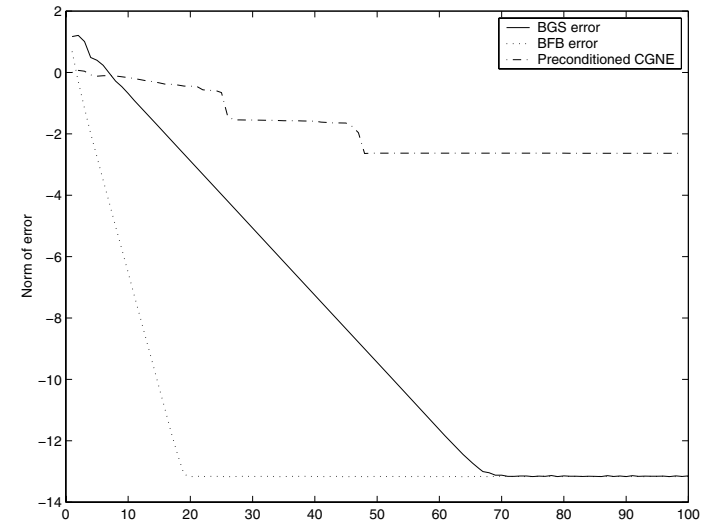

Figure 2: Convergence rates for first example. Solid line (-) BGS method, dotted line (.) BFB, dash-dot (-.) preconditioned CGNE.

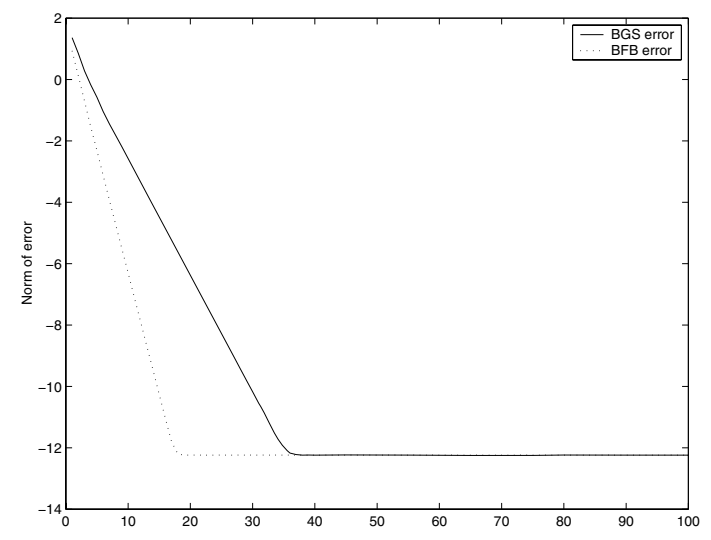

Figure 3: Convergence rates for second example. Solid line (-) BGS method, dotted line (.) BFB.

\section{Acknowledgments}

This publication has emanated from research conducted with financial aid from Science Foundation Ireland.

\section{References}

[1] J. C. West and J. M. Sturm, "On iterative approaches for electromagnetic rough-surface scattering problems", IEEE Trans. AP. Vol. 47 No. 8, August 1999, pp.1281-1288

[2] C. Brennan and D. Bogusevschi, "Convergence analysis for buffered block forward-backward (BBFB) method applied to EFIE", IEEE Intl. Ant. Prop. Symp. July 2006,Albuquerque, U.S.A.

[3] D. A. Kapp and G. S. Brown, "A new numerical method for roughsurface scattering calculations, IEEE Trans. Antennas Propagat., vol. 44, pp. 711721, May 1996.

[4] D. Holliday, L. L. DeRaad, Jr., and G. J. StCyr, "Forwardbackward: A new method for computing low-grazing angle scattering, IEEE Trans. Antennas Propagat., vol. 44, pp. 722729, May 1996.

[5] A. F. Peterson, S. L. Ray and R. Mittra "Computational methods for Electromagnetics", IEEE Press ISBN 0-7803-1122-1.

[6] G. Golub C. van Loan , "Matrix computations", 3rd edition, The Johns Hopkins University Press, London. 